\title{
Reproductive Tract Tissue/Organ Engineering and Regenerative Medicine for Reproduction
}

\author{
Ayman Al-Hendy ${ }^{1} \cdot$ Seung-Yup Ku ${ }^{2}$ \\ Published online: 27 April 2021 \\ (C) Society for Reproductive Investigation 2021
}

In efforts to conquer diseases, decades of research, via their success and failure, have seen both the pros and cons of the current therapeutics and medications. These efforts improved the QoL of many individuals and enhanced our understanding of complex medical conditions by uncovering mechanisms behind the structural and functional aberrations leading to the onset of such diseases. These efforts entail numerous strategies involving exogenous or manipulated materials applied to the human body, which have contributed to the understanding and development of new concepts for human health. Intravenous fluid therapy, for instance, was developed in the 1800 s, and the medical field has never imagined returning to its prior era, given its prevalent use in modern practice. The development of antibiotics in the early 1900s is still deemed one of the greatest advances in the medical history. It has permanently changed the way healthcare provider approaches cases involving infectious agents, albeit rapidly rising levels of bacterial resistance, with a greater certainty of the outcomes. Discovering the structure of DNA and its role in life and developing various molecular techniques enabled larger, exciting projects, namely, the Human Genome Project, which turned the dream of gene therapy into a reality. Although gene therapy saw its shortcoming in the 1990s, it has inspired and continues to show ways of developing novel therapeutic strategies with superior cell source and innovative technologies.

Tissue engineering is defined as technology that incorporates a designed biodegradable scaffold to replace or support

Seung-Yup Ku

jyhsyk@snu.ac.kr

Ayman Al-Hendy

aalhendy@bsd.uchicago.edu

1 Department of Obstetrics and Gynecology, University of Chicago, 5801 S Ellis Ave, Chicago, IL 60612, USA

2 Department of Obstetrics and Gynecology, Seoul National University Hospital, 28 Yonkeun-dong, 110-744, Chongno-gu, Seoul, South Korea the dysfunctional tissue and organ. The realm of tissue engineering has further expanded into the use of stem cells, especially by means of induced pluripotent and organ-specific stem cells that promote cell proliferation, differentiation, and new tissue growth. Cells and bioactive molecules are incorporated into bio-friendly scaffolds to assist their repair of congenital defects, disease, and injury of organs. As the new era of tissue engineering involves advanced multi-genomics and techniques, the necessity to better understand the developmental processes of certain tissues and the onset of diseases becomes more pronounced. The attraction of tissue engineering of certain organs are met with the needs of reproductive science, a synergy that is expected to grow, especially with the emergence of female stem cells; hence, the newly coined term "Reproductive Bioengineering" or "Female Reproductive Tissue Engineering" is aimed to solve the unsolved mysteries of female reproductive tract disorders.

From the perspective of reproductive science, exciting resurgences have occurred through tissue engineering and stem cells. To date, the focus of reproductive sciences has been laid on fertility and reproduction, including assisted reproductive techniques, uterine disorder, pregnancy and recovery, and postmenopausal health. iPSC technologies triggered the generation of female germ cell from stem cells, which was believed to be almost impossible in the adult female. Reproductive bioengineering includes the engineering of the artificial ovary, treatment of intractable uterine dysfunctions such as Asherman's syndrome, and the generation of female gametes. One of the main advances was utilized for the structural abnormality like MRKH syndrome, which was recovered by the tissue-engineered treatment at WFIRM, and its key members shared their latest research on this special issue. Dr. Anthony Atala developed a bioengineered bladder and successfully recovered the function, which was published at NEJM in 1998. This historical study inspired the field of reproductive science as a whole because the bladder is part of the urogenital system with its similarity to some of the female reproductive organs. Dr. James Yoo focused on the 
development of artificial ovaries with engineered somatic cells neighboring oocytes and gained functionality, which has an impact on the infertility of aged women.

These studies expanded the roles of tissue engineering in reproductive sciences, demonstrating how engineered models provide a platform to evaluate and screen the repair and regeneration of reproductive organs after anticancer treatments. These models suggest a possibility of reproductive engineering serving as a future mode of therapeutics in overcoming the current limitations.

This special issue focuses on the various platforms of reproductive engineering, covered in 16 basic and translational research papers. Reviews and original articles on fertility preservation, pre-term birth, and female reproductive organs illustrate the long-term goal and necessity of engineering in reproductive medicine while pointing out the limitations of the current therapeutics. Engineering of female reproductive organs with animal models from small to large pre-clinical models, small molecules with enhanced effects, and 3D models are included in this special issue. The stem cell paracrine effect on the in vitro activation of the pre-antral follicle was presented as an emerging technology as a new alternative source of oocytes. The treatment approach for POI with stem cells is also discussed. The effects of small molecules on engineering platform were demonstrated. Since various types of the hydrogel are used for the ovary and uterus, a team of scholars used a well-known scaffold, chitosan with specific effective molecules for follicle such as melatonin and tretinoin. In another report, oviduct-derived decellularized hydrogel was used for embryo culture. The construction of animal models resembling clinical features is an important one, as it serves as the foundation for evaluating the efficacy of the newly developed therapeutic strategies. An endometrial injury model was introduced that was constructed by transcervical ethanol perfusion along with the limited supply of models for vaginal research. An emerging technology, 3D culture or also widely known as an organoid, in reproductive organs were explored.

We are very proud to present the contents in this special issue and hope to expand the range of our journal, Reproductive Sciences, which aims to cover a wide range of topics in reproductive engineering from basic engineering to clinical issues. Reproduction is traditional, but also amenable for innovation, because of its indispensable connection to our lives confronted with social and medical changes. For the unmet needs of reproductive sciences, we hope this special issue serves as a guide, defining the field of "Reproductive Bioengineering" and paving ways to prospective research which will surely provide strategies for the betterment of women's reproductive health.

Publisher's Note Springer Nature remains neutral with regard to jurisdictional claims in published maps and institutional affiliations. 